\title{
Association of Hyperuricemia With Hypertension In Pakistani Population
}

\author{
Attia Khaliq, Abdul Moueed Tariq, Abdul Latif Khattak, Karamat Ali, Shahzeb Ahmad Satti,
} Hafiz Yasir Rehman

\section{ABSTRACT}

Objective: To find an association between Hyperuricemia and Hypertension in patients presenting in outpatients department for regular check-up.

Study design and Setting: Case Control Study design conducted at Pak Emirates Military Hospital Rawalpindi (PEMH), Medical Outpatient department (OPD) from $4^{\text {th }}$ Feb, 2017 to $4^{\text {th }}$ Aug, 2017.

Methodology: A total of 200 patients, with 100 cases and 100 controls fulfilling the selection criteria were included in the study, which were selected from Medical OPD of PEMH. An informed written consent was taken. The Demographic such as age, gender were obtained. Veriable upon selection into study group, the blood samples were taken and two groups of patients were made on the basis of normal or raised uric acid levels. The Serum Uric Acid (SUA) was measured in the patients. Then blood pressure was measured by using sphygmomanometer by researchers themselves. If BP level was $>140 / 90 \mathrm{mmHg}$ on 2 separate occasions, or patient is already a known case of hypertension, then hypertension was labeled.

Results: The mean age (years) in the study was $54.79+8.96$. There were $136(68 \%)$ male and $64(32 \%)$ female patients. The frequency and percentage of hypertension in cases and control groups was (84\%) and (41\%) patients respectively which was statistically significant with a p-value of 0.000. The Odds ratio was found to be 7.55.

Conclusion: It was concluded that there was an association found between Hyperuricemia and Hypertension.

Key words: Hypertension, Hyperuricemia, Uric Acid.

\section{INTRODUCTION:}

The hypertension is very much prevalent and it affects approximately one-third of world population and is one of the common cause of death and disability. ${ }^{1,2}$ Though the cause of hypertension is not known in most of the patients, SUA(serum uric acid) has been thought to activate the renin - angiotensin system, which results in damage to prerenal blood vessels. ${ }^{3}$ Recently, a new study has shown that the mild hyperuricemia induces the arteriopathy and hypertension and this has brought renewed interest into this hypothesis. ${ }^{4,5}$

- - - - - - - - - - - - - - -

Ittia Khaliq

I FCPS, Department of Medicine,

I Pak Emirates Military Hospital, Rwp Cantt

I Abdul Moueed Tariq

Resident, Department of Medicine,

Combined Military Hospital, Quetta Cantt

I Email: amoueed@hotmail.com

I Abdul Latif Khattak

I Classified Medical Specialist,

Combined Military Hospital, Quetta Cantt

I Karamat Ali

FCPS, Department of Medicine,

PNS Shifa Hospital, Karachi

I

Shahzeb Ahmad Satti

Classified Medical Specialist,

I Combined Military Hospital, Quetta Cantt

I Hafiz Yasir Rehman

I Resident, Department of Medicine,

I Combined Military Hospital, Quetta Cantt

I Received: 10-Apr-2019

Accepted: 03-Mar-2020
The global prevalence of hypertension in adults is around $40 \% .{ }^{6}$ SUA is produced as result of a catabolism of normal purine, which is excreted mostly in urine but also through the gastrointestinal tract. Many studies have shown SUA concentrations $>7 \mathrm{mg} / \mathrm{dL}$ is an independent and one of the other major risk factor associated with hypertension, a reduction in SUA is associated with lowering $\mathrm{BP}^{7,8}$ The mean uric acid levels and number of hyperuricemic subjects were found to be significantly higher in hypertensive cases than normotensive controls. ${ }^{9,10}$ In a study conducted by Redon and colleagues on patients from European countries showed that $25 \%$ of population had hyperuricemia. It was associated with greater prevalence of metabolic syndrome, diabetes mellitus, rate of uncontrolled hypertension in these patients. ${ }^{11}$ In a study conducted by Xiaoyun Lin et al in china concluded that, in males, hyperuricemia was an independent risk factor of hypertension with an adjusted OR of $1.131 .^{12}$ The hyperuricemia was also associated with post partum hypertension as concluded by Caraline C. Mars and colleagues with OR of $2.44 .^{13}$

The younger individuals and women are at higher risk as compared to others. ${ }^{14,15}$ In one of study in 2011, among cases of hyperuricemia, the frequency of hypertension was $36.6 \%$ and among patients of normal serum uric acid level was $18.8 \%$. The difference was found to be significant $(\mathrm{P}<0.01){ }^{16}$

Similarly NHANES 2009-2012 study has found that among cases of hyperuricemia, the frequency of hypertension was $38 \%$ while among patients of normal serum uric acid level was $20 \%$. The difference was found to be significant 
$(\mathrm{P}<0.01) .{ }^{17}$ In a study done in kenya by C.B. Mibey Sylvia and colleagues showed that prevalence of hyperuricemia was $44 \%$ in hypertensive patients; with $37.6 \%$ and $47.3 \%$ in males and females respectively. ${ }^{18}$ In a study by Kuwabara $\mathrm{M}$ et al in Japan showed that the OR for association between hyperuricemia and hypertension were 1.79 in the total study population, 1.58 in men and 1.60 in women. ${ }^{19}$ The regional studies in Pakistan revealed that hyperuricemia was found in $40.3 \%$ and $37.4 \%$ in hypertensive population. ${ }^{20}$

The rationale of this study was to assess the association between Hyperuricemia and Hypertension in patients presenting in outpatient department for regular check-up for other chronic illnesses in our populatin. It has been noticed in previous studies, that hyperuricemia may lead to increased cardiovascular events and accelerate the BP level. Therefor the study was aimed to find the association between Hyperuricemia and Hypertension in patients presenting in outpatients department for regular check-up.

\section{METHODOLOGY:}

This was a case control study done in Medical OPD of PEMH Rawalpindi, from 4th Feb, 2017 to 4th Aug, 2017 with a sample size of 200 cases: 100 cases and 100 controls. The sample size was calculated using $80 \%$ as power of test, with level of significance 5\% and taking $18.8 \%$ as prevalence of hypertension in patients without hyperuricemia and $36.6 \%$ in those patients who were having hyperuricemia. ${ }^{8}$ The cases were selected by non-probability, consecutive sampling technique. An Informed written consent was taken. The Patients with age between 35 to 70 years of either gender, presenting in OPD for regular checkup as follow up cases of various diseases (after applying exclusion criteria) were considered for selection. The patients taking drugs such as thiazide \& loop diuretics, pyrazinamide, Cytotoxic drugs and those Patients with CKD, eclampsia, lymphoma, leukemia were excluded from the study. The demographic variables as age, gender were obtained.

From recruited patients, the blood samples were taken to determine the serum uric acid levels. After obtaining lab results patients were categorized as cases (those with hyperuricemia) and controls(those with normal serum uric acid levels), using the upper limit of normal range for both genders as cut off for hyperuricemia i.e $>7.4 \mathrm{mg} / \mathrm{dl}$ for males and $>5.8 \mathrm{mg} / \mathrm{dl}$ for females. Then blood pressure was measured by using sphygmomanometer by researchers themselves. If BP level was $>140 / 90 \mathrm{mmHg}$ on 2 separate occasions, or patient is already diagnosed case of hypertension on anti hypertensive therapy, then hypertension was labeled.

The Data analyzed in SPSS version 20. The descriptive statistics was calculated for both qualitative and quantitative variables. The quantitative variables like age, blood pressure were analyzed as mean and standard deviation. The qualitative variables such as gender and hypertension were presented as frequency and percentage. The Chi square test was applied to compare hypertension between 2 groups and the Odd ratio was calculated. The effect modifiers such as gender, Diabetes, age, dyslipidemia, History of Ischemic heart disease were controlled by stratification. P-value < 0.05 was taken as statistically significant.

\section{RESULTS:}

A total of 200 patients were enrolled in of the study protocol applying the strict inclusion and exclusion criteria. The two groups of patients were made randomly. The cases consist of those patients who were found to have hyperuricemia whereas patients in control group were without hyperuricemia (normal serum uric acid level). There were 136 (68\%) male and $64(32 \%)$ female patients in our study, as shown in chart No. 01 . The Mean age (years) in the study was $54.79+8.96$ with ranges from 35 to 70 years.

The descriptive statistics blood pressure was measured twice time. The mean systolic Blood pressure in $\mathrm{mmHg}$ at $1^{\text {st }}$ and $2^{\text {nd }}$ reading was $136.75+16.20$ and $136.17+16.02$ respectively whereas mean diastolic Blood pressure in $\mathrm{mmHg}$ at $1^{\text {st }}$ and $2^{\text {nd }}$ reading was $86.32+9.71$ and $85.50+8.73$, as shown in Table No. 01.

The Frequency of hypertension in the study was 125 (62.5\%) whereas $75(37.5 \%)$ patient were without hypertension, as shown in Table No. 02

The objective was to find out the association between hyperuricemia and hypertension in patients presenting in outpatients department for regular check-up. The Frequency and Percentage of hypertension in both the groups was 84 (84\%) and 41 (41\%) patients respectively. The Chi Square test was used to compare hypertension in both the groups. The $\mathrm{P}$ value and odds ratio, as shown in Table No. 03

\section{DISCUSSION:}

The global prevalence of hypertensionin adults 25 years and above was around $26.4 \%$ and this figure is estimated to increase to about $60 \%$ to a total number 1.5 billion in year $2025 .{ }^{6,21}$ In a study conducted in Japan, 43 million individuals were estimated to have high blood pressure with male and female prevalence of $60 \%$ and $45 \%$ respectively.

Figure 1: Gender Distribution

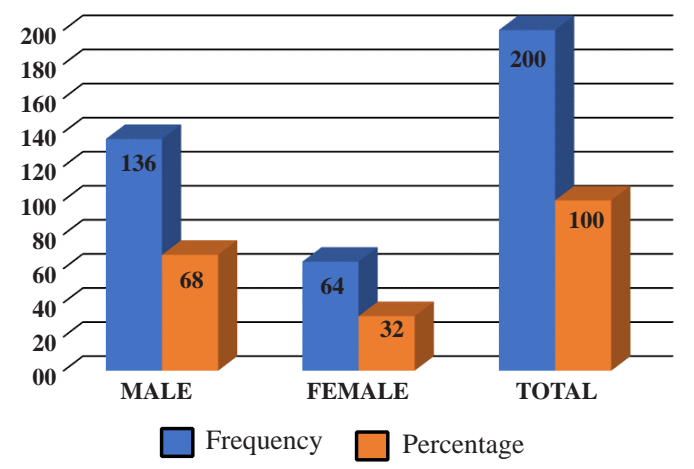


Association of Hyperuricemia With Hypertension In Pakistani Population

Table 1: Descriptive statistics of Blood Pressure (systolic \& diastolic) $1^{\text {st }}$ and $2^{\text {nd }}$ reading

\begin{tabular}{|c|l|c|c|c|}
\hline Reading & & $\mathrm{n}$ & $\begin{array}{c}\text { Mean } \\
(\mathrm{mmHg})\end{array}$ & $\begin{array}{c}\text { Std. } \\
\text { Deviation }\end{array}$ \\
\hline \multirow{2}{*}{$1^{\text {st }}$ reading } & Blood pressure (systolic) & 200 & 136.75 & 16.20 \\
\cline { 2 - 5 } & Blood pressure (diastolic) & 200 & 86.32 & 9.71 \\
\hline \multirow{2}{*}{$2^{\text {nd }}$ reading } & Blood pressure (systolic) & 200 & 136.17 & 16.02 \\
\cline { 2 - 5 } & Blood pressure (diastolic) & 200 & 85.50 & 8.73 \\
\hline
\end{tabular}

Table 2: Frequency \& Percentage of Hypertension

\begin{tabular}{|c|c|c|c|}
\hline \multicolumn{2}{|c|}{} & Frequency & Percentage \\
\hline \multirow{4}{*}{ Hypertension } & No & 75 & $37.5 \%$ \\
\cline { 2 - 4 } & Yes & 125 & $62.5 \%$ \\
\cline { 2 - 4 } & Total & 200 & $100 \%$ \\
\hline
\end{tabular}

Table. 3: Association of Hyperuricemia and Hypertension in both the groups

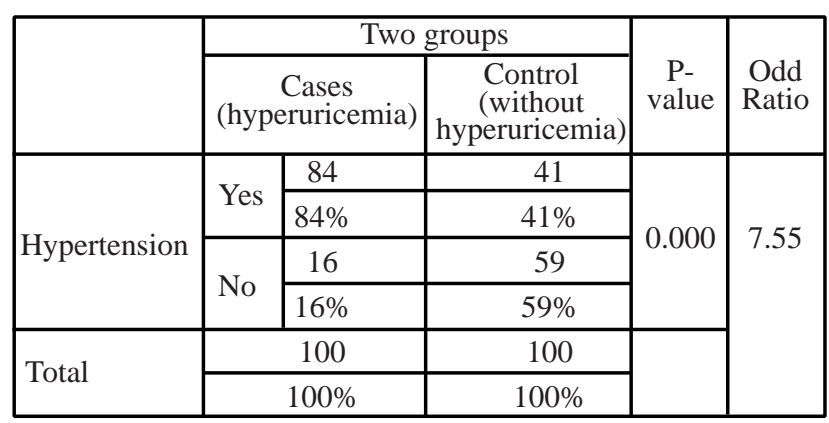

In developing countries one of the most common cause of death is Coronary artery disease. The cardio-vascular diseases has been the cause of more than 17.5 million deaths in year 2012 globally. $^{22}$ The one of a major risk factor for cardiovascular death and morbidity is hypertension. ${ }^{23}$

The Hyperuricemia also results in an increased risk for cardiovascular disease. It is also associated with gout, renal dysfunction and the metabolic syndrome. The association between hypertension and hyperuricemia is being studied worldwide in recent years. The hyperuricemia plays a vital role in the development of hypertension, and association between the two, has been studies previous in many studies. The association between hyperuricemia and CV diseases in patients with hypertension is also being reported. ${ }^{24}$ Many studies have shown that drugs that reduce serum uric acid levels ,also reduce BP in patients with hyperuricemia. ${ }^{25,26}$ There are various mechanisms involved by which increased blood pressure occurs in patients with elevated uric acid. ${ }^{27}$ Our study result showed that there were $84 \%$ of patients with hyperyricemia, which were hypertensive while only $41 \%$ without hyperuricemia had increased blood pressure. There was a strong association between hypeuricemia and hypertension with Odds ratio of 7.55. In a study done by Yokokawa et al, Hyperuricemia was 1.79 times more common in men with hypertension in comparison with those without than hypertension and almost 6 times more prevalent in hypertensive women with odds ratio of 5.92, while odds ratio in our study population was $7.55 .{ }^{8}$

In research done by Poudel et al, the mean SUA levels and number of patients with hyperuricemia was, significantly higher in hypertensive group, than those without hypertension $(28.8 \%$ vs $13.7 \%)$ with Odds ratio of $2.55 .^{28}$ Shrivastav et al showed that the mean SUA levels and hyperuricemiac patients were significantly higher in newly diagnosed cases of essential hypertension as compared to prehypertensive or normotensive controls (37.33\% vs $14 \%)$. In our study, percentage of hypertension in patients with hyperuricemia was $84 \%$ and without hyperuricemia, it was $41 \%$ respectively. In a study in Pakistan, that the SUA levels were significantly increased in patients with hypertension $(6.51 \pm 1.45 \mathrm{mg} / \mathrm{dl})$ than those without hypertension $(4.72 \pm 1.83 \mathrm{mg} / \mathrm{dl}) .{ }^{29} \mathrm{In}$ another study by Bâ Hamidou Oumar et al, the prevalence of hyperuricemia was found to about $66 \%$ in hypertensive patients, while it was $84 \%$ in our study. ${ }^{30}$ Therefore, blood pressure should be monitored regularly in patients with hyperuricemia or gout. Patients with Hypertension should be monitored for increased serum uric acid levels. This will help us treat raised uric acid levels to reduce the burden of patients with hypertension as well reduce the risk of other cardiovascular diseases.

\section{CONCLUSION:}

It was concluded that the association was found between Hyperuricemia and Hypertension.

\section{REFRENCES:}

1. Grayson PC, Kim SY, LaValley M, Choi HK. Hyperuricemia and incident hypertension: a systematic review and metaanalysis. Arthritis care \& research. 2011;63(1):102-10.

2. Wang J, Qin T, Chen J, Li Y, Wang L, Huang H, et al. Hyperuricemia and risk of incident hypertension: a systematic review and meta-analysis of observational studies. PloS one. 2014;9(12):e114259.

3. Borghi C, Rosei EA, Bardin T, Dawson J, Dominiczak A, Kielstein JT, et al. Serum uric acid and the risk of cardiovascular and renal disease. Journal of hypertension. 2015;33(9):1729 41.

4. Tsai C-W, Lin S-Y, Kuo C-C, Huang C-C. Serum Uric Acid and Progression of Kidney Disease: A Longitudinal Analysis and Mini-Review. PloS one. 2017;12(1):e0170393.

5. Takahashi A, Takabatake Y, Isaka Y, Saitoh T, Yoshimori T. Hyperuricemia-induced inflammasome and kidney diseases. Nephrology Dialysis Transplantation. 2015;31(6):890-6.

6. Mills KT, Bundy JD, Kelly TN, Reed JE, Kearney PM, Reynolds K, et al. Global Burden of Hypertension: Analysis of Population-based Studies from 89 Countries. Journal of Hypertension. 2015;33:e2.

7. Kanbay M, Jensen T, Solak Y, Le M, Roncal-Jimenez C, Rivard C, et al. Uric acid in metabolic syndrome: from an innocent bystander to a central player. European journal of internal medicine. 2016;29:3-8. 
8. Yokokawa H, Fukuda H, Suzuki A, Fujibayashi K, Naito T, Uehara Y, et al. Association between serum uric acid levels/hyperuricemia and hypertension among 85,286 Japanese workers. The Journal of Clinical Hypertension. 2016;18(1):539.

9. Mallat SG, Al Kattar S, Tanios BY, Jurjus A. Hyperuricemia, hypertension, and chronic kidney disease: an emerging association. Current hypertension reports. 2016;18(10):74.

10. Geraci G, Mulè G, Mogavero M, Geraci C, Nardi E, Cottone S. Association Between Uric Acid and Renal Hemodynamics: Pathophysiological Implications for Renal Damage in Hypertensive Patients. The Journal of Clinical Hypertension. 2016;18(10):1007-14.

11. Redon P, Maloberti A, Facchetti R, Redon J, Lurbe E, Bombelli $\mathrm{M}$, et al. Gender-related differences in serum uric acid in treated hypertensive patients from central and east European countries: findings from the Blood Pressure control rate and CArdiovascular Risk profilE study. Journal of Hypertension. 2019;37(2):380-8.

12. Lin X, Wang X, Li X, Song L, Meng Z, Yang Q, et al. Genderand Age-Specific Differences in the Association of Hyperuricemia and Hypertension: A Cross-Sectional Study. International Journal of Endocrinology. 2019;2019:9.

13. Marrs CC, Rahman M, Dixon L, Olson G. The association of hyperuricemia and immediate postpartum hypertension in women without a diagnosis of chronic hypertension. Hypertension in Pregnancy. 2018;37(3):126-30.

14. Chen Y-Y, Kao T-W, Yang H-F, Chou C-W, Wu C-J, Lai C$\mathrm{H}$, et al. The association of uric acid with the risk of metabolic syndrome, arterial hypertension or diabetes in young subjectsAn observational study. Clinica Chimica Acta. 2018;478:6873.

15. Saladini F, Mos L, Fania C, Garavelli G, Casiglia E, Palatini P. Regular physical activity prevents development of hypertension in young people with hyperuricemia. Journal of hypertension. 2017;35(5):994-1001.

16. Yokokawa H, Fukuda H, Suzuki A, Fujibayashi K, Naito T, Uehara Y, et al. Association Between Serum Uric Acid Levels/Hyperuricemia and Hypertension Among 85,286 Japanese Workers. Journal of clinical hypertension (Greenwich, Conn). 2016;18(1):53-9.

17. Krishnan E. Interaction of inflammation, hyperuricemia, and the prevalence of hypertension among adults free of metabolic syndrome: NHANES 2009-2010. Journal of the American Heart Association. 2014;3(2):e000157.

18. Sylvia CM, Some F, Kimaiyo S, Kwobah C, Oyoo G. Prevalence and risk factors for hyperuricemia among patients with hypertension at Moi Teaching and Referral Hospital, Eldoret, Kenya. African Journal of Rheumatology. 2018;6(1):39.
19. Kuwabara M, Niwa K, Nishi Y, Mizuno A, Asano T, Masuda $\mathrm{K}$, et al. Relationship between serum uric acid levels and hypertension among Japanese individuals not treated for hyperuricemia and hypertension. Hypertension research : official journal of the Japanese Society of Hypertension. 2014;37(8):785-9.

20. Ahmed N, Anwar W, Waqas H. Obesity, hyperlipidemia, and hyperuraecemia in young and old hypertensive patients. Journal of Ayub Medical College Abbottabad. 2009;21(4):536.

21. Kearney PM, Whelton M, Reynolds K, Muntner P, Whelton PK, He J. Global burden of hypertension: analysis of worldwide data. The lancet. 2005;365(9455):217-23.

22. Goyal A, Kahlon P, Jain D, Soni RK, Gulati R, Chhabra ST, et al. Trend in prevalence of coronary artery disease and risk factors over two decades in rural Punjab. Heart Asia. 2017;9(2):e010938-e.

23. Patel P, Ordunez P, DiPette D, Escobar MC, Hassell T, Wyss F, et al. Improved Blood Pressure Control to Reduce Cardiovascular Disease Morbidity and Mortality: The Standardized Hypertension Treatment and Prevention Project. The Journal of Clinical Hypertension. 2016;18(12):1284-94.

24. Acevedo A, Benavides J, Chowdhury M, Lopez M, Pena L, Montenegro A, et al. Hyperuricemia and Cardiovascular Disease in Patients with Hypertension. Connecticut medicine. 2016;80(2):85-90.

25. Gois PHF, de Moraes Souza ER. Pharmacotherapy for hyperuricemia in hypertensive patients. Cochrane Database of Systematic Reviews. 2017(4).

26. Madero M, Castellanos FER, Jalal D, Villalobos-Martín M, Salazar J, Vazquez-Rangel A, et al. A pilot study on the impact of a low fructose diet and allopurinol on clinic blood pressure among overweight and prehypertensive subjects: a randomized placebo controlled trial. Journal of the American Society of Hypertension. 2015;9(11):837-44

27. Abeles AM. Hyperuricemia, gout, and cardiovascular disease: an update. Current rheumatology reports. 2015;17(3):13.

28. Poudel B, Yadav BK, Kumar A, Jha B, Raut KB. Serum uric acid level in newly diagnosed essential hypertension in a Nepalese population: a hospital based cross sectional study. Asian Pacific journal of tropical biomedicine. 2014;4(1):5964.

29. Jawed S, Khawaja TF, Sultan MA, Ahmad S. The effect of essential hypertension on serum uric acid level. Biomedica (Lahore). 2005:98-102.

30. Oumar BH, Ichaka M, Kéita MA, Adama D, Bella DI, Souleymane C, et al. Prevalence of Hyperuricemia (HU) in Arterial Hypertension. Science. 2015;4(4):76-9. 